\title{
Collision parameters governing water delivery and water loss in early planetary systems
}

\author{
Thomas I. Maindl and Rudolf Dvorak \\ Universitätssternwarte, University of Vienna \\ A-1180, Vienna, Austria \\ email: thomas.maindl@univie.ac.at
}

\begin{abstract}
We investigate the distribution of encounter velocities and impact angles describing collisions in the habitable zone of the early planetary system. Here we present a catalogue of collision characteristics for a particular mass ratio of the colliding bodies and seven different planetesimal masses ranging from a tenth of Ceres' mass to 10 times the mass of the Moon. We show that there are virtually no collisions with impact speeds lower than the surface escape velocity and a similar velocity-impact angle distribution for different planetesimal masses if velocities are normalized using the escape velocity. An additional perturbing Jupiter-like object distorts the collision velocity and impact picture in the sense that grazing impacts at higher velocities are promoted if the perturber's orbit is close to the habitable zone whereas a more distant perturber has more the effect of a mere widening of the velocity dispersion.
\end{abstract}

Keywords. solar system: formation, celestial mechanics, methods: n-body simulations

\section{Introduction}

The goal of this study is to quantify and characterize the number of collisions between planetesimals in the early planetary system. Depending on the masses involved, we directly determine the velocity and the direction of the collisions between these bodies. In this approach we do not yet intend to accumulate them to larger ones; this is another task which is actually already in the stage of computations. The next step - this is the core of our overall project - is modeling the collisions in detail with our SPH code, which will allow us to track water content and explain water delivery processes by collisions and impacts (cf. Dvorak et al. 2012).

As dynamical model we use full n-body integrations of the Sun and a ring of planetesimals in the habitable zone. The bodies' initial orbital elements are uniformly distributed with $0.9<a<1.1 \mathrm{AU}$ (semi-major axis), $e<0.1$ (eccentricity), and $i<1^{\circ}$ (inclination), respectively. Additionally, we check the influence of a gas giant of $1 \mathrm{M}_{4}$ in different distances to the planetesimals which acts as a perturber in one particular scenario.

The scenarios differ in the chosen mass for the bodies which varies from $5 \cdot 10^{-11} \mathrm{M}_{\odot} \approx$

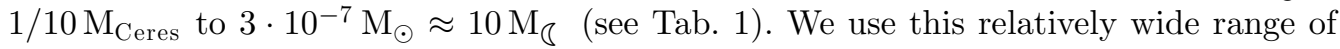
masses to see its influence on the collision velocities and impact angles of the two bodies. Each scenario is integrated for $10^{6}$ years and includes 750 planetesimals.

In point mass-based n-body simulations we assume a collision to happen if two objects get as close as $R_{\text {imp }}=R_{P}+R_{T}$ with $R_{P}$ and $R_{T}$ denoting the respective radii of spherical bodies represented by the point masses. In each scenario this distance is significantly smaller than the Hill radius (cf. Tab. 1).

Therefore - along with our interest in the statistical distribution of impact angles and velocities - we treat collisions as two-body problems of total mass $M_{\text {tot }}=2 m=M_{P}+M_{T}$ 
Table 1. N-body simulation scenarios $-m$ is the planetesimal mass, $R_{\text {imp }}$ denotes the mutual distance of the barycenters upon impact for $C_{\mathrm{P}}=0.3, C_{\mathrm{T}}=0, M_{\mathrm{P}} / M_{\mathrm{tot}}=0.1, r_{\mathrm{Hill}}$ the Hill radius at $1 \mathrm{AU}$ and zero eccentricity, and $v_{\text {esc }}$ the target's surface escape velocity (see text).

\begin{tabular}{llccrr}
\hline \hline Scenario & $m\left[\mathrm{M}_{\odot}\right]$ & $m[\mathrm{~kg}]$ & $R_{\text {imp }}\left[10^{6} \mathrm{~m}\right]$ & $r_{\text {Hill }}\left[10^{6} \mathrm{~m}\right]$ & $v_{\text {esc }}[\mathrm{m} / \mathrm{s}]$ \\
\hline Ce10 & $5 \cdot 10^{-11}$ & $9.95 \cdot 10^{19}$ & 0.3918 & 38 & 247 \\
Ce & $5 \cdot 10^{-10}$ & $9.95 \cdot 10^{20}$ & 0.8440 & 82 & 532 \\
M10 & $3 \cdot 10^{-9}$ & $5.97 \cdot 10^{21}$ & 1.534 & 150 & 967 \\
M3 & $1 \cdot 10^{-8}$ & $1.99 \cdot 10^{22}$ & 2.291 & 223 & 1444 \\
M & $3 \cdot 10^{-8}$ & $5.97 \cdot 10^{22}$ & 3.304 & 322 & 2083 \\
3M & $1 \cdot 10^{-7}$ & $1.99 \cdot 10^{23}$ & 4.936 & 481 & 3112 \\
10M & $3 \cdot 10^{-7}$ & $5.97 \cdot 10^{23}$ & 7.119 & 694 & 4488 \\
\hline
\end{tabular}
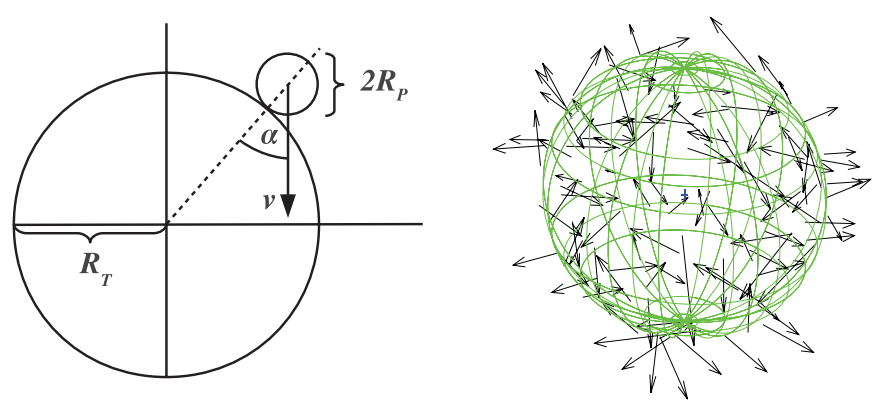

Figure 1. Left: impact geometry with impact angle $\alpha$, impact velocity vector $\mathbf{v}$, and projectile and target radii $R_{P}$ and $R_{T}$, respectively.

Right: schematic view of impact velocities in the $3 \mathrm{M}$ scenario. The arrows show the negative impact velocity for better visibility. The majority of impacts occurs from low inclination orbits.

with projectile and target masses $M_{P}$ and $M_{T}$, respectively. For given $M_{P}$ and given mean mean densities, $R_{\mathrm{P}}, R_{\mathrm{T}}$, and $R_{\mathrm{imp}}$ can be computed. As we are interested in water delivery by impacts we assume a solid basalt core with a layer or mantle of water ice, similar to the current models of Ceres (cf. Thomas et al. 2005).

We get the following relation for the radius of a body of mass $M_{\{P, T\}}$ consisting of basalt and a water ice shell (densities $\rho_{\mathrm{b}}, \rho_{\mathrm{i}}$ ) with a certain ice mass-fraction $C_{\{P, T\}}$ :

$$
R_{\{P, T\}}^{3}=\left[C_{\{P, T\}}+\left(1-C_{\{P, T\}}\right) \frac{\rho_{\mathrm{i}}}{\rho_{\mathrm{b}}}\right] M_{\{P, T\}} \frac{3}{4 \pi} \frac{1}{\rho_{\mathrm{i}}} .
$$

Keeping the future SPH-based simulations in mind we adopt the same values for the densities of ice and basalt as in Maindl et al. (2013): $\rho_{\mathrm{i}}=917 \mathrm{~kg} / \mathrm{m}^{3}$ and $\rho_{\mathrm{b}}=2,700 \mathrm{~kg} / \mathrm{m}^{3}$.

As a representative parameter set for collisions of interest for water delivery we choose a scenario with $M_{\mathrm{P}} / M_{\text {tot }}=0.1$ and respective water contents $C_{\mathrm{P}}=30 \%$ and $C_{\mathrm{T}}=0$.

\section{Results}

In each scenario multiple collisions occur. As the initial "disk" of planetesimals is not considerably widened during the integration interval most of the impacts are close to the ecliptic (Fig. 1, right). As expected bigger masses correspond to bigger mutual perturbations and hence a larger number of collisions. The impact velocities show a relatively wide spread with a well defined lower boundary of about the target's surface escape velocity $v_{\text {esc }}$ and an upper bound that varies between about 2.5 and $11.5 v_{\text {esc }}$. Fig. 2 shows scatter plots of velocities versus impact angles (Fig. 1, left defines the impact angle). 

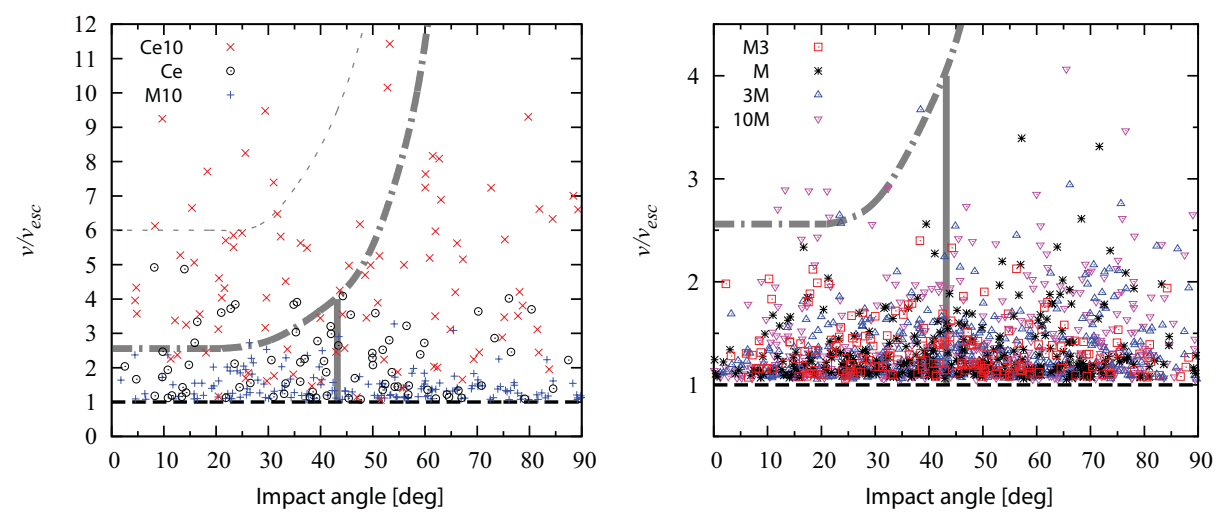

Figure 2. Collision velocities normalized by the target's surface escape velocities versus impact angle for the different scenarios. Note that high velocities only occur in the Ce10 scenario. The lines refer to the boundaries of different collision outcomes as given in Leinhardt \& Stewart (2012): net erosion to the target above the dot-dashed curve, below it partial accretion to the left and hit-and-run events to the right of the thick vertical line. There is no collision in the area of perfect merging (below the dashed line, $v / v_{\text {esc }} \lesssim 1$ ). The dotted curve in the left diagram denotes the critical disruption velocity for half the total mass remaining in the largest remnant.

Table 2. Total number of collision events $N_{\text {tot }}$ for the different scenarios broken down to number of collisions $N$, impact velocities (mean $\bar{v}$ and median $m_{v}$ ), and their standard deviations $\sigma$ for three impact angle intervals. An impact angle of $0^{\circ}$ corresponds to a head-on collision. Velocity units are $\mathrm{km} / \mathrm{s}$.

\begin{tabular}{lrrrrrrrrrrrrrr}
\hline \hline \multirow{2}{*}{ Scenario } & \multicolumn{1}{c}{$N_{\text {tot }}$} & $N$ & \multicolumn{1}{c}{$0^{\circ}-30^{\circ}$} & \multicolumn{1}{c}{$30^{\circ}-60^{\circ}$} & \multicolumn{4}{c}{$60^{\circ}-90^{\circ}$} \\
Ce10 & 94 & 31 & 1.11 & 1.00 & 0.54 & 31 & 1.02 & 0.96 & 0.62 & 32 & 1.20 & 1.16 & 0.54 \\
Ce & 79 & 23 & 1.22 & 1.03 & 0.64 & 41 & 1.16 & 1.15 & 0.47 & 15 & 1.07 & 0.74 & 0.57 \\
M10 & 158 & 45 & 1.51 & 1.42 & 0.44 & 61 & 1.42 & 1.28 & 0.44 & 52 & 1.34 & 1.28 & 0.34 \\
M3 & 195 & 62 & 1.96 & 1.83 & 0.41 & 91 & 1.92 & 1.76 & 0.38 & 42 & 1.87 & 1.84 & 0.29 \\
M & 303 & 80 & 2.65 & 2.50 & 0.47 & 137 & 2.75 & 2.51 & 0.67 & 86 & 2.88 & 2.54 & 0.82 \\
3M & 449 & 126 & 3.93 & 3.64 & 0.78 & 197 & 4.11 & 3.84 & 0.94 & 126 & 4.26 & 3.76 & 1.13 \\
10M & 483 & 134 & 5.99 & 5.14 & 1.84 & 201 & 6.03 & 5.40 & 1.56 & 148 & 6.51 & 5.76 & 2.15 \\
\hline
\end{tabular}

The results of all seven considered scenarios in absolute velocity units are summarized in Tab. 2. The standard deviations along with the relatively large deviations of the mean and median values confirm the wide spread of impact velocities as observed in the scatter plots, especially for large-mass scenarios.

Tab. 3 and the plot in Fig. 3 show how different perturbing "Jupiters" effect the collision velocities in the Ce scenario. Especially a gas giant very close to the habitable zone significantly increases the spread in the velocities due to highly perturbed orbits. Also, hit-and-run collisions happen more often in that case. The significantly larger spread in the velocities may also increase the number of destructive collisions - an effect that decreases towards a "real" Jupiter at larger distance $a_{\text {pert }}=5.2 \mathrm{AU}$.

\section{Conclusions and further research}

In our n-body calculations we confirm typical collision velocities in the early planetary system that range from the target's surface escape velocity $v_{\text {esc }}$ up to a few times $v_{\text {esc }}$ depending on the mass of the planetesimals. There is a clear tendency to collision velocities 
Table 3. The effect of a perturbing body in the Ce scenario. The perturbing body's mass is $1 \mathrm{M}_{4}$, its initial semi axis $a_{\text {pert }}$. The other symbols and units are the same as in Tab. 2.

\begin{tabular}{lccccccccccccccc}
\hline & \multicolumn{1}{c}{$a_{\text {pert }}$} & \multicolumn{1}{c}{$0^{\circ}-30^{\circ}$} & \multicolumn{4}{c}{$30^{\circ}-60^{\circ}$} & \multicolumn{4}{c}{$60^{\circ}-90^{\circ}$} \\
Scenario & {$[\mathrm{AU}]$} & $N_{\text {tot }}$ & $N$ & $\bar{v}$ & $m_{v}$ & $\sigma$ & $N$ & $\bar{v}$ & $m_{v}$ & $\sigma$ & $N$ & $\bar{v}$ & $m_{v}$ & $\sigma$ \\
\hline $\mathrm{Ce}$ & 1.6 & 80 & 17 & 2.50 & 2.28 & 1.38 & 27 & 2.62 & 2.23 & 1.46 & 36 & 2.71 & 2.27 & 1.60 \\
$\mathrm{Ce}$ & 2.6 & 80 & 16 & 1.75 & 1.73 & 0.99 & 37 & 2.03 & 1.87 & 1.05 & 27 & 2.05 & 1.93 & 1.26 \\
$\mathrm{Ce}$ & 5.2 & 71 & 19 & 1.03 & 0.84 & 0.45 & 35 & 1.39 & 1.30 & 0.74 & 17 & 1.68 & 1.41 & 0.94 \\
\hline
\end{tabular}

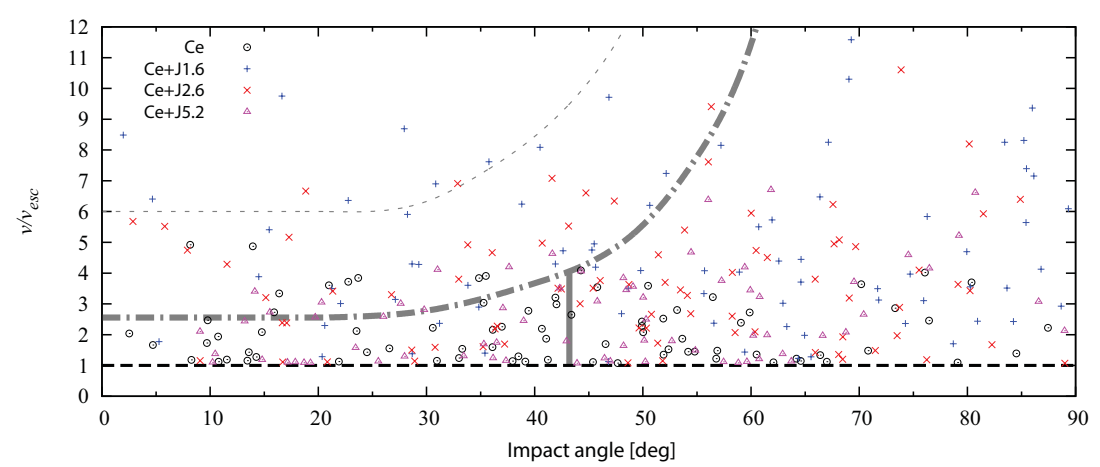

Figure 3. The Ce scenario without and with a perturbing gas giant of $1 \mathrm{M}_{4}$ placed at 1.6, 2.6, and 5.2 AU from the Sun (denoted by Ce+J1.6, Ce+J2.6, and Ce+J5.2, respectively). The lines and symbols have the same meaning as in Fig. 2.

$v / v_{\text {esc }}$ closer to 1 for heavier objects which may be explained by the two-body acceleration during the impact event dominating the initial velocity dispersion of the bodies for larger masses. The influence of perturbing bodies such as gas giants is significant in case of the perturber's orbit close to the planetesimals in the sense of a widened distribution of impact velocities and a tendency to promote hit-and-run collisions at high impact angles.

In the future, we will use collision velocities and angles corresponding to the partial accretion and hit-and-run scenarios in Figs. 2 and 3 as input to analyzing water transport via collisions. We will use our 3D SPH code that - among other features - includes elastoplastic material modeling, brittle failure, self-gravity, and first order consistency fixes. It is introduced in Schäfer (2005) and Maindl et al. (2013) and is still developed further.

\section{Acknowledgements}

The authors wish to thank C. Schäfer and R. Speith for jump-starting us on collisions of solid bodies and SPH as well as for numerous fruitful discussions. We also thank Á. Süli and D. Bancelin for discussing and critically reviewing our n-body results. This research is produced as part of the FWF Austrian Science Fund project S 11603-N16.

\section{References}

Dvorak, R., Eggl, S., Süli, Á., Sándor, Z., Galiazzo, M., \& Pilat-Lohinger, E. 2012, AIP Conf. Proc., 1468, 137

Leinhardt, Z. M. \& Stewart, S. T. 2012, ApJ, 745, 79

Maindl, T. I., Schäfer, C., Speith, R., Süli, A., Forgács-Dajka, E., \& Dvorak, R. 2013, AN, 334, 9,996

Schäfer, C. 2005, Dissertation, Eberhard-Karls-Universität Tübingen, Germany

Thomas, P. C., Parker, J. Wm., McFadden, L. A., Russell, C. T., Stern, S. A., Sykes, M. V., \& Young, E. F. 2005, Nature, 437, 224 